DEPÓSITO LEGAL ppi $201502 Z U 4666$ Esta publicación científica en formato digital es continuidad de la revista impresa

ISSN 0041-8811

\title{
Revista de la Universidad del Tulia
}

Fundada en 1947 par el Dr. Jesús Emrique Lossada

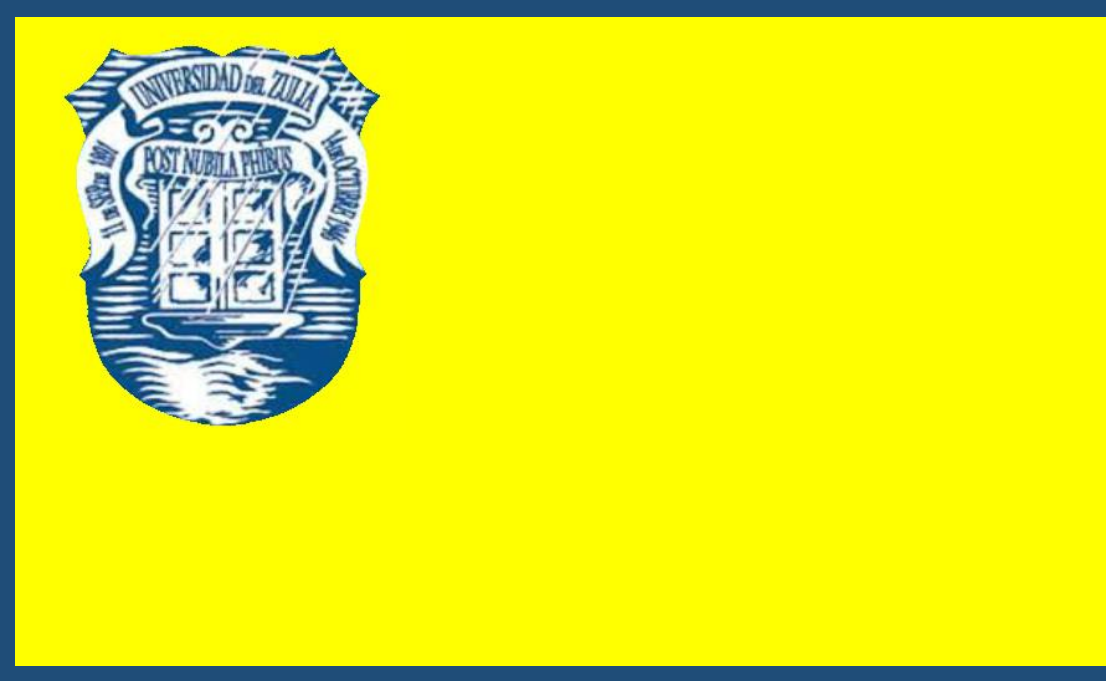

\section{Ciencias}

Exactas

Naturales

y de la Salud

\section{Aกัต 12 No 33 \\ Mayo - Agosto 2021 \\ Tercera ipoca \\ Maracaibo-Venezucla}




\title{
Desconexión digital de los profesores investigadores del área de Ciencias de la Salud
}

\author{
María del Pilar Quezada Castro* \\ María del Pilar Castro Arellano ** \\ Juan Manuel Oliva Núñez*** \\ Carlos Ignacio Gallo Aguila **** \\ Guillermo Alexander Quezada Castro*****
}

RESUMEN

El artículo trata sobre la desconexión digital como derecho del docente universitario que asume el rol de investigador en las ciencias de la salud. El objetivo del estudio consistió en determinar la importancia de la desconexión digital para el bienestar del docente investigador universitario peruano del área de ciencias de la salud. Esta investigación fue planteada desde el método fenomenológico hermenéutico, el mismo que permitió obtener como resultados que la familia y la investigación científica son pilares esenciales para el docente investigador. Se concluyó que la desconexión digital es un derecho del profesional a su descanso diario y tranquilidad que protege la salud mental.

PALABRAS CLAVE: Contrato de trabajo; desconexión digital; gestión; investigador científico.

* Docente en la Universidad Tecnológica del Perú. Coordinadora Académica de las Escuelas de Derecho y Psicología de la Universidad Tecnológica del Perú sede Piura, https://orcid.org/0000-0002-1012-570X Email: c20853@utp.edu.pe

** Docente en la Universidad Alas Peruanas y Universidad Tecnológica del Perú, sede Piura, https://orcid.org/0000-0002-6661-9928 Email: maripili_1728_19@hotmail.com

*** Coordinador de la oficina de Investigación, Innovación y Emprendimiento de la Universidad Alas Peruanas, Piura. Docente a tiempo completo en la Universidad Alas Peruanas, Perú - filial Piura. https://orcid.org/0000-0001-9319-3689 Email: j_oliva@doc.uap.edu.pe

**** Docente en la Universidad César Vallejo. https://orcid.org/0000-0003-1382-0545. Email: cgalloa@gmail.com

***** Docente a tiempo completo en la Universidad Alas Peruanas, Perú -filial Piura. https://orcid.org/0000-0002-4868-1664. E-mail: guillermoquezadacastro@hotmail.com

Recibido: 01/02/2021

Aceptado: 05/04/2021 


\section{Digital disconnection of research professors in the Health Sciences area}

ABSTRACT

The article deals with digital disconnection as a right of the university teacher who assumes the role of researcher in the health sciences. The objective of the study was to determine the importance of digital disconnection for the well-being of the Peruvian university research professor in the area of health sciences. This research was proposed from the hermeneutical phenomenological method, the same one that allowed obtaining as results that the family and scientific research are essential pillars for the research teacher. It was concluded that digital disconnection is a professional's right to daily rest and peace of mind that protects mental health.

KEYWORDS: Work contract; digital disconnection; management; scientific researcher.

Introducción

La virtualidad de la enseñanza se extendió a partir de la pandemia denominada COVID19, lo cual exigió la aplicación de las tecnologías de información y comunicación en beneficio de la continuidad del servicio educativo.

A nivel universitario, se aprecia que el docente se adaptó al uso cotidiano de los entornos virtuales de aprendizaje, y para ello empleó en su hogar los requerimientos tecnológicos mínimos que hicieran viable el proceso de enseñanza hacia sus estudiantes, específicamente, en cuanto a pedagogía y metodología.

La investigación y docencia constituyen la esencia de la universidad; para ambas se requiere dedicación, responsabilidad y tiempo, que será destinado a la enseñanza y aprendizaje de nuevos conocimientos de una asignatura o especialidad.

El tiempo vinculado hacia una actividad podría ser la diferencia en la actuación de un docente investigador, quien asume el reto de formar habilidades y competencias investigativas en sus estudiantes, las cuales serán comprobadas en el ejercicio profesional. 
REVISTA DE LA UNIVERSIDAD DEL ZULIA. 3a época. Año 12 N³3, 2021

María del Pilar Quezada et al.// Desconexión digital de los profesores investigadores ... 196-208

DOI: http://dx.doi.org/10.46925//rdluz.33.14

En este contexto, se identifica como objetivo determinar la importancia de la desconexión digital para el bienestar del docente investigador universitario peruano del área de ciencias de la salud.

$\mathrm{Al}$ respecto, se debe tener en cuenta que la universidad, como en toda organización, se vincula -entre otros temas-, atendiendo a la jerarquía y cumplimiento de un horario de trabajo, siendo que después del mismo, el trabajador tiene derecho a gozar de su descanso y tiempo libre. Sin embargo, existen empleadores que se comunican fuera de la jornada laboral, contrariando el derecho a la desconexión digital que ostenta el trabajador (Pérez, 2020), es decir, se exige mayor conectividad a las plataformas digitales por encima de la jornada ordinaria de trabajo (Taléns, 2019), situación que podría afectar los ámbitos familiar y social.

En el mundo digital se prioriza la reducción de espacios físicos (Molina, 2017a) y el respeto por la forma de vida laboral, familiar y personal (Serrano, 2019); es por ello que los mercados globales son más competitivos (Naranjo, 2017), al no vincularse exclusivamente por el registro de la jornada de trabajo (Arrieta, 2019), situación que se encuentra amparada por el derecho a la intimidad (Requena, 2020), el cual es sustento del derecho a la desconexión digital.

La desconexión digital se reconoce como aquella situación que supera la voluntad del trabajador (Vallecillo, 2020), a quien se le obliga asumir una conexión tecnológica permanente con el entorno laboral (Gutiérrez, 2020), sin tener en cuenta el tiempo de descanso, permisos y vacaciones que ello representa (Megino \& Lanzadera, 2020), lo cual afecta la esfera privada y la salud de los trabajadores (Pérez, 2019; Andrusova et al., 2020).

En el contexto actual, se aprecia que los empleadores establecen una subordinación extra laboral, la misma que contiene actividades ajenas al horario de trabajo materializado en el contrato suscrito por las partes; por lo tanto, aquellas actividades extra laborales vulneran el derecho del trabajador a estar desconectado digitalmente, máxime si constituyen el sometimiento a una evaluación constante (Pazos, 2020); de tal forma que el concepto de tiempo libre estaría aparentemente limitado y restringido.

$\mathrm{Al}$ respecto, se debe tener en cuenta que el desarrollo profesional del docente representa la configuración de experiencias y oportunidades (Henríquez, Castillo \& Rodríguez, 2019); por 
lo tanto, no es recomendable centralizar su actuación solamente en la asignación de una calificación hacia el estudiante (Ortiz, Santos \& Marín, 2020), antes bien, corresponderá a la universidad desarrollar las competencias tanto de docentes como de estudiantes (Cejas, et al., 2020), permitiendo la cohesión entre la tecnología y la humanidad; esta combinación incidirá en la formación de investigadores (Montoya, 2019; Vaganova, 2019).

En tal sentido, es importante precisar que cada docente se caracteriza por un hábito tecnológico que le incentiva a navegar por internet (Castillejos, Torres \& Lagunes, 2016), a través de dispositivos digitales que incorpora paulatinamente en su rutina cotidiana (Flores, Rojas \& Straubhaar, 2017; Ibujés \& Franco, 2019). Sin embargo, se identifica un vacío al no brindarse la garantía del respeto por la vida privada en una sociedad digital (Molina, 2017 b), la misma que preconcibe a las Tecnologías de información y comunicación - TIC como una herramienta que pretende mejorar la calidad de vida a nivel laboral (Cialti, 2017).

Es un deber de todo docente realizar investigación, actividad destinada a producir cambios en las universidades (Martelo, Jaramillo \& Ospino, 2018), las mismas que se presentan ante la sociedad con una nueva identidad global (Rendueles, 2016) y trabajo de producción científica (Chachaima-Mar et al., 2018). En tal sentido, la selección de los docentes investigadores está a cargo de la oficina de talento humano (Barros \& Turpo, 2020), por lo tanto, debe promover la formación en investigación en las universidades (Barros \& Turpo, 2017), de tal forma que se consolide como una práctica habitual en el ejercicio docente (Palencia et al., 2019).

El rol del docente como investigador comprende, entre otros temas, la difusión de los resultados de investigación (Fernández et al., 2016); para lograrlo se debe publicar en bases de datos internacionales (De Souza, De Filippo \& Sanz, 2020), es decir, se requiere que el docente a dedicación exclusiva desarrolle su producción científica (Chalela-Naffah \& Rodríguez, 2020), solo así es factible adquirir visibilidad como institución universitaria (Altbach \& De Wit, 2018).

En este sentido, Fuster (2019I) consideró que la fenomenología hermenéutica se presenta como un método que "está orientado a la descripción e interpretación de las estructuras fundamentales de la experiencia vivida, al reconocimiento del significado del valor 
REVISTA DE LA UNIVERSIDAD DEL ZULIA. 3a época. Año 12 N³3, 2021

María del Pilar Quezada et al.// Desconexión digital de los profesores investigadores ... 196-208

DOI: http://dx.doi.org/10.46925//rdluz.33.14

pedagógico de esta experiencia”. (p.207), siendo que este método podría ser aplicado en el presente estudio.

Finalmente, el rol del docente como investigador está relacionado con el salario que percibe, evitando que recurra a otras actividades que generen ingresos económicos (Méndez \& Vera, 2015); las universidades requieren que se promuevan actividades con énfasis en investigación (Paredes, Casanova \& Naranjo, 2019), que se creen cimientos para una cultura de investigación en los docentes universitarios (Yangali et al., 2020) y para lograrlo se requiere sobre todo que se garantice el derecho a la desconexión digital, el mismo que se presenta como una garantía en la formación de competencias investigativas en el docente (Gutiérrez, 2020; Montes \& Mendoza, 2018).

\section{Metodología}

La presente investigación se diseñó desde el paradigma naturalista, enfoque cualitativo y método fenomenológico hermenéutico.

Se elaboró el instrumento para la realización de la entrevista, la misma que contenía siete preguntas que fueron validadas mediante juicio de 3 expertos, lo que permitió realizar las respectivas modificaciones. Asimismo, se consideró necesario aplicar el Coeficiente de Validez de Contenido Total, obteniéndose como resultado 0,821; ello complementó y otorgó la validez al instrumento utilizado.

Se entrevistó a 15 docentes con vínculo laboral vigente en cinco universidades peruanas, los cuales fueron seleccionados con los siguientes criterios: a) Formación académica en investigación científica, b) Formación académica en redacción científica en ciencias de la salud y c) Publicación de un artículo en revista indizada, ello en el último año.

Según Fuster (2019) el método fenomenológico hermenéutico "está orientado a la descripción e interpretación de las estructuras fundamentales de la experiencia vivida, al reconocimiento del significado del valor pedagógico de esta experiencia". Agrega que comprende 4 fases: primera fase (preconceptos del tema de investigación), segunda fase (entrevista), tercera fase (reflexión) y cuarta fase (integración). 
En el presente estudio, se utilizó el método fenomenológico hermenéutico para recabar la información de campo y se aplicó en la primera fase seleccionando a los posibles participantes, aunque la invitación fue realizada a 30 docentes, solo participaron activamente 15 docentes universitarios, a quienes se les establecieron preguntas relacionadas con la desconexión digital y el rol del investigador en ciencias de la salud; las respuestas fueron resumidas y consignadas como resultados. Posteriormente, en la segunda fase se tomaron apuntes de las experiencias personales de los docentes participantes, evitando solicitar explicaciones causales al respecto. En la tercera fase, el equipo investigador consideró necesario trasmitir la esencia captada de las entrevistas realizadas. En la última fase, se estableció como característica del grupo estudiado que la labor del docente investigador del área de ciencias de la salud debe estar acompaada de la desconexión digital.

\section{Resultados y discusión}

De la aplicación del método fenomenológico hermenéutico, el equipo investigador obtuvo los siguientes resultados, los cuales se redactaron atendiendo a las cuatro fases descritas en la metodología del presente estudio.

En la primera fase se incluyó la entrevista a los 15 docentes investigadores seleccionados, a quienes se les planteó la siguiente interrogante: iel docente investigador debe estar desconectado digitalmente?, obteniéndose en resumen las siguientes respuestas:

Entrevistado l: Sí, el docente universitario que investiga debe dedicarse exclusivamente a ello, de tal forma, que se debe evitar cualquier atadura que limite su libertad y tranquilidad.

Entrevistado 2: Sí, el docente investigador debe aprender a gestionar su tiempo, específicamente, en temas de investigación.

Entrevistado 3: Sí, la desconexión digital es un derecho de todo docente universitario, el no reconocerlo sería obstaculizar la producción científica.

Entrevistado 4: Sí, la investigación debe estar separada de la gestión académica producto de reuniones. 
REVISTA DE LA UNIVERSIDAD DEL ZULIA. 3a época. Año 12 N³3, 2021

María del Pilar Quezada et al.// Desconexión digital de los profesores investigadores ... 196-208

DOI: http://dx.doi.org/10.46925//rdluz.33.14

Entrevistado 5: Sí, respetar la desconexión digital es un deber de las autoridades universitarias.

Entrevistado 6: Sí, el docente investigador debe estar desconectado digitalmente porque ello permite centralizar su atención hacia el incremento de la producción científica.

Entrevistado 7: Sí, la universidad tiene la responsabilidad en respetar el descanso y premiar el incentivo en cuanto a la producción científica.

Entrevistado 8: Sí, la desconexión digital evita la sobrecarga de trabajo académico al docente universitario.

Entrevistado 9: Sí, el docente universitario debe dedicarse a la enseñanza producto de la investigación realizada.

Entrevistado 10: Sí, se debe priorizar la investigación en lugar de actividades de gestión académica.

Entrevistado 1l: Sí, el docente investigador debe estar desconectado digitalmente, ello contribuye a su concentración de su rol en la universidad.

Entrevistado 12: Sí, la desconexión digital es un derecho de todo docente universitario.

Entrevistado 13: Sí, respetar la desconexión digital podría reducir el estrés laboral del docente universitario.

Entrevistado 14: Sí, la desconexión digital permitiría el descanso del docente e integración familiar.

Entrevistado 15: Sí, el docente investigador es un ser humano y como tal no es justo ni necesario que se pretenda esclavizarlo digitalmente.

La segunda fase permitió recabar información de las experiencias personales de los 15 docentes participantes, a quienes se les preguntó mediante entrevista su vivencia entorno a la desconexión digital y su rol como investigadores universitarios; las respuestas obtenidas esn esta fase, se dividen en dos grupos: 7 docentes afirmaron que la desconexión digital representa un obstáculo a su producción científica y 8 docentes expresaron que la desconexión digital 
afecta su espacio familiar. Con ello se aprecia que tanto la familia como la investigación científica son pilares indispensables para ejercer la docencia en el contexto actual.

En la tercera fase, se centró por reflexionar respecto a los dos pilares advertidos en la fase anterior, es decir, la familia y la investigación científica; de lo cual se obtuvo como resultado que la esencia del docente investigador en ciencias de la salud es en orden prioritario la familia, porque es el hogar un espacio íntimo e integrador de relaciones humanas y es en mérito a ello que el docente procura asumir retos relacionados con la producción científica.

En la cuarta y última fase, el equipo investigador percibe que la característica del grupo estudiado se sustenta en su relación familiar, por lo tanto, cualquier intento que perturbe la misma fuera del horario de trabajo origina intromisión, tal como sucede al exigirse respeto por el derecho a la desconexión digital, lo cual debe ser considerado por el empleador.

Asimismo, de las cuatro fases obtenidas se reconocen las siguientes discusiones:

Respecto a la prioridad de atender a la familia: Esta discusión se deriva al analizar las respuestas, de las 15 entrevistas se advierte que el 100\% de los docentes seleccionados consideraron que la integración familiar representa el primer elemento que afectaría la conectividad permanente, ello es comprensible en la medida que el ser humano protege lo que más anhela, siendo su núcleo familiar el principal motivo para su desenvolvimiento a nivel personal y profesional; por lo tanto, este podría ser el primer elemento que deben tener en cuenta las autoridades universitarias, con la finalidad de incentivar mayor producción científica en los docentes, aspecto que se recomienda analizar con mayor detenimiento en una futura investigación.

Respecto al derecho de descanso diario: Esta discusión está relacionada con la tranquilidad y paz que debe percibir el docente universitario que realiza actividad de investigación, de lo contrario, esta misma situación podría convertirse en un cuadro de estrés laboral, hecho que podría afectar el derecho de salud del personal contratado y podría perjudicar el servicio educativo de excelencia.

Respecto a la formación de investigadores: Esta discusión se deriva al asumir que la generación de competencias investigativas transita por un proceso de formación, el mismo que 
REVISTA DE LA UNIVERSIDAD DEL ZULIA. 3a época. Año 12 N³3, 2021

María del Pilar Quezada et al.// Desconexión digital de los profesores investigadores ... 196-208

DOI: http://dx.doi.org/10.46925//rdluz.33.14

expresa estudio, error y éxito; sin embargo, el elemento característico de lo descrito está relacionado con el tiempo, es decir, la forma cómo cada docente investigador distribuye su tiempo podría ser la diferencia entre ser investigador o no.

Respecto a la producción científica: Esta discusión se deriva al analizar las respuestas de los 15 docentes seleccionados, apreciándose que se prioriza la labor de investigador en lugar del docente universitario; siendo ello así, se aprecia que la producción científica constituye el elemento diferenciador que es necesario incentivar a nivel de pregrado y posgrado.

\section{Conclusiones}

El docente investigador peruano del área de ciencias de la salud debe estar desconectado digitalmente, toda vez que es su derecho como profesional gestionar su tiempo, distribuirlo entre la gestión académica, enseñanza en aula e investigación; estas actividades no deben interferir su espacio familiar o su descanso diario.

La desconexión digital debe ser promovida por parte de las autoridades universitarias con la finalidad de incentivar mayor producción científica en los docentes.

El incumplimiento del horario de trabajo podría originar situaciones de estrés laboral, la misma que podría afectar la salud mental del docente investigador.

La universidad debe promover la formación de investigadores, quienes deberán asumir el ejercicio de dicho rol en un ambiente de libertad y tranquilidad.

El docente universitario debe ser protegido de cualquer interferencia que origine la desconcentración en su rol como investigador.

Conflictos de intereses

Los autores declaran no tener conflictos de intereses.

\section{Referencias}

Acosta, A. C., Jiménez, L. K., Pullido, E. G. \& Redondo (2019). Estrés ocupacional y evaluación de desempeño en docentes del departamento del Cesar, Colombia. Revista Encuentros, 17(1), 2433. 
REVISTA DE LA UNIVERSIDAD DEL ZULIA. 3a época. Año 12 N³3, 2021

María del Pilar Quezada et al.// Desconexión digital de los profesores investigadores ... 196-208

DOI: http://dx.doi.org/10.46925//rdluz.33.14

Altbach, P. \& De Wit, H. (2018). Se está publicando demasiada investigación académica. Educación superior internacional, (96), 2-3

Andrusova, I.V.; Smirnova, Z.V.; Chelnokova, E.A.; Nikeitseva, O.N.; Tsaregorodtseva, A.N. (2020). Health-saving technologies in teacher's activity, Revista de la Universidad del Zulia, 11 (31), 461-472. DOI: https://doi.org/10.46925//rdluz.31.29

Arrieta, F. J. (2019). La desconexión digital y el registro de la jornada diaria en España como mecanismos para garantizar el descanso, la salud y el bienestar de los trabajadores digitales a distancia. Revista Lan Harremanak, 42, 89-126.

Barros, C., \& Turpo, O. (2020). La formación en investigación y su incidencia en la producción científica del profesorado de educación de una universidad pública de Ecuador. Publicaciones, 50(2), 167-185.

Barros, C. \& Turpo, O. (2017). La formación en el desarrollo del docente investigador: una revisión sistemática. Revista Espacios, 38(45), 11-20.

Barrachina, M., Crespo, C. \& Ripoll, V. M. (2020). Limitations of rule 23 on the allocation of costs of teaching and research staff in Spanish public universities. Cuadernos de Gestión, 20(2), 7596.

Castillejos, B., Torres, C. A. \& Lagunes, A. (2016). La seguridad en las competencias digitales de los millennials. Revista Apertura, 8(2), 54-69.

Cejas, M., Mendoza, D., Alban, C. \& Frías, E. (2020). Caracterización del perfil de las competencias laborales en el docente universitario. Revista Orbis, 15(45), 23-37.

Chachaima-Mar, J. E. , Fernández-Guzmán, D. \& Atamari-Anahui, N. (2018). Publicación científica de docentes de una escuela de medicina peruana: frecuencia y características asociadas. Revista Educación Médica, 20(2), 2-9.

Chalela-Naffah, S. \& Rodríguez, D. (2020). Caracterización del profesorado con perfil investigador en universidades colombianas. Revista Electrónica Educare, 24(3), 1-20.

Cialti, P. (2017). El Derecho a la Desconexión en Francia: ¿Más de lo que parece? Revista Temas Laborales, 137, 163-181.

Cruz, C., Soto, O. \& Morales, P. (2019). Estrés docente: causas y repercusiones laborales. Magazine de las ciencias. Revista de Investigacion e Innovación, 4(4), 145-156.

De Souza, C., De Filippo, D. \& Sanz, E. (2020). El papel de la internacionalización de la Educación Superior en la producción científica brasileña. Ensaio: Avaliação e Políticas Públicas em Educação, 28 (108), 784-810.

Fernández, J., Molfino, V. \& Ochoviet, C. (2016). Rol Docente del Investigador en Matemática Educativa: un Ejemplo en un Curso de Posgrado para Profesores del Nivel Superior. Revista Bolema, Río Claro (SP), 30(55), 808-829. 
REVISTA DE LA UNIVERSIDAD DEL ZULIA. 3a época. Año 12 N³3, 2021

María del Pilar Quezada et al.// Desconexión digital de los profesores investigadores ... 196-208

DOI: http://dx.doi.org/10.46925//rdluz.33.14

Flores, M., Rojas, V. \& Straubhaar, J. (2017). Brecha digital en la frontera de EE.UU. - México: Un estudio de caso multigeneracional en Laredo, Texas. Revista Estudios Fronterizos, 18(37), 18-40.

Fuster, D. (2019). Investigación cualitativa: Método fenomenológico hermenéutico. Revista Propósitos y Representaciones, 7(1), 201-229.

Gutiérrez, D. (2020). La desconexión digital de los trabajadores. Reflexiones a propósito de su calificación como derecho y su instrumentación. Revista de Internet, Derecho y Política, 31, 1-13.

Henríquez, M. A., Castillo, H. P. \& Rodríguez, C. G. (2019). Valoración de ámbitos formativos del docente universitario de la carrera Educación en la Universidad de Los Andres - Táchira. Revista Electrónica Formación y Calidad Educativa, 7(3), 119-133.

Ibujés, J.M. \& Franco, A. (2019). Uso de las TIC y relación con los Objetivos de Desarrollo Sostenible en Ecuador, Revista RETOS, 9 (17), 37-53. https://doi.org/10.17163/ret.nl7.2019.03

Martelo, R., Jaramillo, J. M. \& Ospino, M. (2018). Producción científica de docentes universitarios y estrategias para aumentarla mediante series de tiempo y MULTIPOL. Revista Espacios, 39(16), 1-10.

Megino, D. \& Lanzadera, E. (2020). El derecho a la desconexión digital: delimitación y análisis, Aplicación práctica en la Administración Pública. Revista Vasca de Gestión de personas y Organizaciones Públicas, 18, 64-91.

Méndez, J. \& Vera, L. (2015). Salarios, incentivos y producción intelectual docente en la universidad pública en Colombia. Revista Apuntes Cenes, 34(60), 95-130.

Mesurado, B. \& Laudadío, J. (2019). Experiencia profesional, capital psicológico y engagement. Su relación con el burnout en docentes univeristarios. Propósitos y Representaciones, 7(3), 12-40.

Molina, C. (2017a). Jornada laboral y tecnologías de la información: "desconexión digital", garantía del derecho al descanso. Revista Temas Laborales, 138, 249-283.

Molina, C. (2017b). El tiempo de los derechos en un mundo digital: iexiste un nuevo "Derecho Humano a la Desconexión" de los trabajadores fuera de jornad? Revista de la Facultad de Derecho de México, 67 (269), 891-919.

Montes, I. \& Mendoza, P. (2018). Docencia e Investigación en Colombia desde la Perspectiva del Capitalismo Académico. Revista Archivos Analíticos de Políticas Educativas, 26(40), 1-33.

Montoya, W. C. (2019). Actividad investigativa en el docente universitario. Revista Horizontes, 3(9), 15-32.

Naranjo, L. D. (2017). Vicisitudes del nuevo derecho a la desconexión digital: Un análisis desde la base del derecho laboral. Revista Saber, Ciencia Y Libertad, 12(2), 49-57.

Ortiz, E., Santos, J. M. \& Marín, S. (2020). Evaluación continua en la enseñanza universitaria de la contabilidad. Revista de Investigación Educativa, 38(1), 109-129. 
REVISTA DE LA UNIVERSIDAD DEL ZULIA. 3a época. Año 12 N³3, 2021

María del Pilar Quezada et al.// Desconexión digital de los profesores investigadores ... 196-208

DOI: http://dx.doi.org/10.46925//rdluz.33.14

Palencia, V., Villagrá, S. \& Rubia, B. (2019). Posibilidades y tensiones del docente-investigador en la escuela. El caso de Argentina, Brasil, Colombia y México. Revista Fuentes, 21(1), 115-134.

Paredes, I., Casanavova, I. \& Naranjo, M. (2019). Transversalidad curricular como vía para el desarrollo de competencias investigativas. Revista Opción, 35(89-2), 599-632.

Pazos, A. (2020). El trabajo autónomo y los sistemas reputacionales. Temas laborales: Revista andaluza de trabajo y bienestar social, 151, 123-138.

Pérez, A. I. (2019). La desconexión digital en España: iun nuevo derecho laboral? Anuario Jurídico y Económico Escurialense, LII, 101-124.

Pérez, F. (2020). Derecho de los trabajadores a la desconexión digital: mail on holiday. Revista del Instituto de Ciencias Jurídicas de Puebla, México, 14(45), 257-275.

Rendueles, C. (2016). La ciudadanía digital. ¿Ágora aumentada o individualismo postmaterialista? Revista Latinoamericana de Tecnología Educativa. Revista Latinoamericana de Tecnología Educatia, 15(2), 15-24.

Requena, O. (2020). Derecho a la desconexión digital: un estudio de la negociación colectiva. Lex Social: Revista de Derechos Sociales, 10(2), 541-560.

Rosenhaum, F. (2020). El "derecho" a la desconexión con especial énfasis en el sistema jurídico uruguayo. Derecho \& Sociedad, 53, 111-122.

Santaella, S. R. (2019). El docente universitario como promotor de la educación mediada por las tecnologías de información y comunicación libre. Revista In Crescendo, 9(3), 399-415.

Serrano, M. (2019). "Always on". Propuestas para la efectividad del derecho a la desconexión digital en el marco de la economía 4.0. Revista Internacional y Comparada de Relaciones Laborales y Deecho del Empleo, 7(2), 164-191.

Tacca, D. R., Tacca, A. L. \& Alva, M. A. (2019). Estrategias neurodidácticas, satisfacción y rendimiento académico en estudiantes universitarios. Revista Cuadernos de Investigación Educativa, $10(2), 15-32$.

Tacca, D. R. \& Tacca, A. L. (2019). Factores de riesgos psicosociales y estrés percibido en docentes universitarios. Propósitos y Representaciones, 7(3), 323-353.

Taléns, E. E. (2019). El derecho a la desconexión digital en el ámbito laboral. Revista Vasca de gestión de personas y organizaciones públicas, 17, 150-161.

Trigueros, R. \& Navarro, N. (2019). La influencia del docente sobre la motivación, las estrategias de aprendizaje, pensamiento crítico y rendimiento académico de los estudiantes de secundaria en el área de Educación Física. Psychology, Society, \& Education, 11(1), 137-150. 
REVISTA DE LA UNIVERSIDAD DEL ZULIA. 3a época. Año 12 N³3, 2021

María del Pilar Quezada et al.// Desconexión digital de los profesores investigadores ... 196-208

DOI: http://dx.doi.org/10.46925//rdluz.33.14

Vaganova, O. (2019). Organization of practical classes in a higher educational institution using modern educational technologies. Amazonia Investiga, 8 (23), 81-86. Retrieved from https:/amazoniainvestiga.info/index.php/amazonia/article/view/850

Vallecillo, M. R. (2020). El derecho a la desconexión digital: perspectiva comparada y riesgos asociados. Revista Internacional y Comparada de Relaciones Laborales y Derecho del Empleo, 8(1), 210-238.

Vallejo, A. B. (2020). El papel del docente universitario en la formación de estudiantes investigadores desde la etapa inicial. Revista de Educación Superior, 34(2), 1-20.

Vásquez-Villanueva, S., Vásquez, S. A., Vásquez, C. A., Alania, R., Díaz-Pinedo, M. \& Gonzáles, M. (2020). El perfil del docente investigador: hacia sus dimensiones y fortalecimiento. Revista Apuntes Universitarios, 10 (4), 69-88.

Yangali, J., Vásquez, M., Huaita, D. \& Luza, F. (2020). Cultura de investigación y competencias investigativas de docentes universitarios del sur de Lima. Revista Venezolana de Gerencia, 25(91), 1159-1179. 